\title{
Competitive Molecular and Dissociative Hydrogen Chemisorption on Size Selected Doubly Rhodium Doped Aluminum Clusters
}

\author{
Jan Vanbuel ${ }^{1}(1) \cdot$ Mei-ye Jia ${ }^{1} \cdot$ Piero Ferrari $^{1}\left(\mathbb{D} \cdot\right.$ Sandy Gewinner $^{2} \cdot$ Wieland Schöllkopf $^{2} \cdot$ Minh Tho Nguyen $^{3}$. \\ André Fielicke $^{2,4}\left(\mathbb{D} \cdot\right.$ Ewald Janssens $^{1}$ (i)
}

\begin{abstract}
The interaction of hydrogen with $\mathrm{Al}_{n} \mathrm{Rh}_{2}{ }^{+}(n=10-13)$ clusters is studied by mass spectrometry and infrared multiple photon dissociation (IRMPD) spectroscopy. Comparing the IRMPD spectra with predictions obtained using density functional theory calculations allows for the identification of the hydrogen binding geometry. For $n=10$ and 11, a single $\mathrm{H}_{2}$ molecule binds dissociatively, whereas for $n=12$ and 13, it adsorbs molecularly. Upon adsorption of a second $\mathrm{H}_{2}$ to $\mathrm{Al}_{12} \mathrm{Rh}_{2}{ }^{+}$, both hydrogen molecules dissociate. Theoretical calculations suggest that the molecular adsorption for $n=12$ and 13 is not due to kinetic impediment of the hydrogenation reaction by an activation barrier, but due to a higher binding energy of the molecularly adsorbed hydrogen-cluster complex. Inspection of the highest occupied molecular orbitals shows that the hydrogen molecule initially forms a strongly bound Kubas complex with the $\mathrm{Al}_{11-13} \mathrm{Rh}_{2}^{+}$clusters, whereas it only binds weakly with $\mathrm{Al}_{10} \mathrm{Rh}_{2}{ }^{+}$.
\end{abstract}

Keywords Hydrogen storage $\cdot$ Metal clusters $\cdot$ Mass spectrometry $\cdot$ IR spectroscopy $\cdot$ Density functional theory calculations $\cdot$ Ion-molecule reactions

\section{Introduction}

Despite its popularity as a propellant in the aerospace industry for several decades [1], the use of hydrogen for our everyday energy supply remains limited. One of the main hurdles is the storage of hydrogen, and its volumetric energy density (in gaseous form about 3000 times lower than that of gasoline [2]) needs to be increased to be considered attractive for mobile applications. Although this can be achieved

Electronic supplementary material The online version of this article (https://doi.org/10.1007/s11244-017-0878-x) contains supplementary material, which is available to authorized users.

Ewald Janssens

ewald.janssens@kuleuven.be

1 Laboratory of Solid State Physics \& Magnetism, KU Leuven, Celestijnenlaan 200D, 3001 Leuven, Belgium

2 Fritz-Haber-Institut der Max-Planck-Gesellschaft, Faradayweg 4-6, 14195 Berlin, Germany

3 Department of Chemistry, KU Leuven, Celestijnenlaan 200F, 3001 Leuven, Belgium

4 Institut für Optik und Atomare Physik, TU Berlin, Hardenbergstraße 36, 10623 Berlin, Germany by compression or liquefaction, these methods entail safety and cost-efficiency issues. Another way to achieve a higher volumetric density is to store hydrogen chemically in the form of metal hydrides, such as $\mathrm{MgH}_{2}$ and $\mathrm{NaAlH}_{4}[3,4]$. Despite the fact that both materials have a high volumetric and gravimetric hydrogen density, their (de)hydrogenation kinetics are too slow for practical purposes [4-6]. A promising venue to alter these slow kinetics is by making use of tailored nano-structured materials that have unique properties compared to their bulk counterparts $[7,8]$. To study the unique properties of those tailored materials, nanoclusters, whose catalytic properties can change by adding or removing a single atom $[9,10]$, are of particular interest. From a more fundamental point of view, clusters provide excellent model systems for reaction kinetics and thermodynamics and the characterization of catalytic active sites [11, 12]. A well-known example is the catalytic activity of small gold clusters in the combustion of CO [13]. A more recent gas phase study suggests that the reduced $\mathrm{CO}$ poisoning of Mo and $\mathrm{Nb}$ doped $\mathrm{Pt}$ catalysts can be explained by a weakening of the CO-Pt bond by dopant induced charge transfer [14]. Furthermore, the countable constituents of atomic clusters make the calculation of their physical and chemical properties computationally feasible for quantum chemistry 
methods such as density functional theory (DFT) and abinitio wave-function-based approaches [15].

Clusters of aluminum, like their bulk counterparts, are, with exception of $\mathrm{Al}_{6,7}{ }^{0,+}$, unreactive towards hydrogen due to a high activation barrier [16, 17]. Doping with transition metals, however, considerably lowers this barrier for aluminum surfaces [18] and a combined experimental and theoretical study on Ti doped $\mathrm{Al}$ [111] suggests that the catalytic active site consists of two neighboring Ti atoms [19]. Similarly, several experimental investigations [20-22] show the enhancing effect of transition metal doping on the reactivity of aluminum clusters, which is also supported by theoretical computations [23]. Although there is a consensus that the enhancement of the reactivity is a local effect caused by the more localized nature of the d-electrons of the transition metal dopant [20, 24], it is not known whether the hydrogen remains molecularly bound, either by van der Waals forces, electrostatically [25], or through the Kubas interaction [26], or if it dissociates upon adsorption; and if so, whether there is spillover from the dopant atom to the aluminum framework or if the hydrogen remains bound to the dopant. In this work, we combine time-of-flight mass spectrometry and infrared multiple photon dissociation spectroscopy (IRMPD) and density functional theory (DFT) calculations to address both questions for size-selected cationic doubly rhodium doped aluminum clusters.

\section{Methodology}

\subsection{Experimental}

Bimetallic rhodium-aluminum clusters are produced in a dual laser ablation source described in more detail elsewhere [27]. In short, the second harmonic $(\lambda=532 \mathrm{~nm})$ light of two $\mathrm{Nd}$ :YAG lasers is focused onto two slowly rotating metal rods, thereby evaporating the material and creating a hot plasma. A short pulse of $\mathrm{He}$ carrier gas with a backing pressure of 5 bar serves to cool the plasma and initiates cluster formation. The clusters are assumed to be thermalized by the carrier gas with the source, which is kept at room temperature. Hydrogen gas at a backing pressure of $1 \mathrm{bar}$ is injected through a separate valve into the source to avoid dissociation of the hydrogen in the ablation plasma, and ensuring that the clusters react with molecular hydrogen. Expansion from the high-pressure region of the source into the vacuum generates a molecular beam of clusters. A 2-mm skimmer is used to select the central part of this molecular beam containing both neutral and charged clusters. The cationic cluster distribution is extracted into an orthogonal time-of-flight mass spectrometer.

The infrared multiple photon dissociation spectra are obtained by scanning the light of a free electron laser [28,
29] in the $800-2100 \mathrm{~cm}^{-1}$ range $\left(P_{\text {avg }}=50 \mathrm{~mJ}\right.$ in a $\sim 10 \mu \mathrm{s}$ long pulse, $10-15 \mathrm{~cm}^{-1}$ FWHM bandwidth) and focusing it onto a 1-mm aperture through which the clusters pass before entering the extraction zone of the mass spectrometer. When the infrared light is in resonance with one of the IR active vibrational modes of the cluster, one or multiple photons can be adsorbed, thereby heating up the cluster through internal vibrational redistribution (IVR) [30]. If enough photons are adsorbed, the internal energy of the cluster reaches the dissociation limit and its abundance in the mass spectrum will deplete. An example of such a depletion in a mass spectrum can be found in Fig. 1. Note that although it is possible that the hydrogen dissociates upon adsorption, depletion in the mass spectra is seen to always occur by loss of $\mathrm{H}_{2}$ (or $2 \mathrm{H}$ ) and not atomic hydrogen. Energetically, it is more favorable to lose both hydrogen atoms and form $\mathrm{a}_{2}$ bond $(\mathrm{BE}=4.48 \mathrm{eV}$ [31]), than to lose a single one: typical $\mathrm{Al}_{n}-\mathrm{H}$ binding energies are $\geq 2 \mathrm{eV}$ [32], whereas calculated $\mathrm{Al}_{n} \mathrm{TM}-\mathrm{H}_{2}$ binding energies all lie below $1 \mathrm{eV}[22,23]$. The IRMPD cross section $\sigma$ of a cluster at a specific wavelength $\nu$ can be calculated as

$\sigma(v)=-\frac{\log \left(I / I_{0}\right)}{P(v)}$

with $I$ and $I_{0}$ the abundance of the cluster-hydrogen complex in the mass spectrum with and without FEL respectively, and $P(\nu)$ the energy per pulse at that wavelength. A complicating factor in the analysis of the IRMPD spectra is the possibility of competing ingrowth and depletion when multiple hydrogen molecules are adsorbed onto the cluster. For most clusters, however, the abundance of the hydrogenated clusters decreases monotonically with the number of adsorbed hydrogen molecules, i.e. $I\left(\mathrm{Al}_{n} \mathrm{Rh}_{2} \mathrm{H}_{2}^{+}\right)>I\left(\mathrm{Al}_{n} \mathrm{Rh}_{2} \mathrm{H}_{4}^{+}\right)>\cdots>I\left(\mathrm{Al}_{n} \mathrm{Rh}_{2} \mathrm{H}_{2 p}{ }^{+}\right)$,

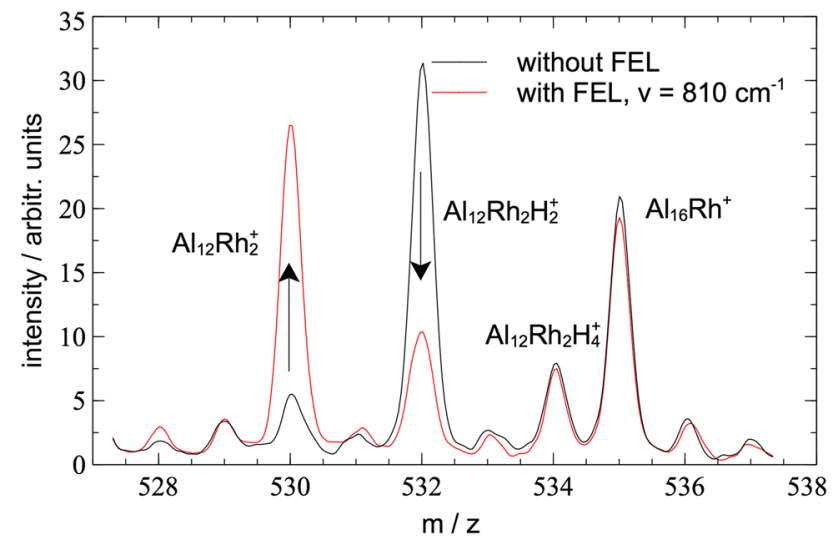

Fig. 1 Part of a mass spectrum with and without FEL light. At irradiation with IR photons of $810 \mathrm{~cm}^{-1}$, the abundance of $\mathrm{Al}_{12} \mathrm{Rh}_{2} \mathrm{H}_{2}^{+}$ decreases, corresponding to an increase in the abundance of $\mathrm{Al}_{12} \mathrm{Rh}_{2}{ }^{+}$ 
and hence the effect of competing channels on the depletion spectra is rather limited.

\subsection{Computational}

DFT methods were used to determine the structures and properties of the clusters considered. An unbiased search for the low-energy structures of $\mathrm{Al}_{n} \mathrm{Rh}_{2}{ }^{+}(n=10-13)$ and their hydrogenated counterparts $\mathrm{Al}_{n} \mathrm{Rh}_{2} \mathrm{H}_{2,4}{ }^{+}$was performed with the aid of the CALYPSO program [33]. Here, we adopted the global version of CALYPSO's particle swarm optimization (PSO) search algorithm to explore the potential energy surface (PES) for each cluster size and cluster-hydrogen complex. For a given cluster, more than 1000 structures were generated and their electronic energies were computed using a low-level quantum chemical method (PBE, LanL2DZ) Of these structures, about 30 lower-lying isomers were selected for further geometry optimization to determine the lowestenergy structure. The relaxed optimization was performed with Gaussian 09, without symmetry constraints, in a range of spin multiplicities. Besides searching with CALYPSO, we have also manually built various initial geometry structures for $\mathrm{Al}_{n} \mathrm{Rh}_{2}{ }^{+}(n=10-13)$ clusters based on pure rhodium and aluminum counterparts with the same number of atoms. Additionally, we constructed a large number of structures for $\mathrm{Al}_{n} \mathrm{Rh}_{2} \mathrm{H}_{2,4}{ }^{+}(n=10-13)$ clusters by adding atomic or molecular hydrogen to the lower energy $\mathrm{Al}_{n} \mathrm{Rh}_{2}{ }^{+}$isomers at different sites.

The generalized gradient approximated (GGA) exchange-correlation functional as devised by Perdew-Burke-Ernzerhof (PBE) [34] was chosen. It is reported that this functional outperforms hybrid counterparts when applied to neutral and low charged binuclear transitionmetal complexes [35]. The adsorption of hydrogen, oxygen, and nitrogen on related clusters $\mathrm{Rh}_{n-1} \mathrm{X}(n=2-5, \mathrm{X}=3 d$, $4 d$ atoms) has systematically been investigated [36] using this functional. The unrestricted formalism was used for open-shell species. The relevance of dispersion forces on the argon and hydrogen was assessed by reoptimization of the lowest energy isomers at the PBE-GD3BJ level, in which dispersion is included through Grimme's D3 scheme and Becke-Johnson damping (PBE-GD3BJ) [37].

The Stuttgart/Dresden (SDD) basis set and the corresponding effective core potential (ECP) [38] was employed for $\mathrm{Rh}$, and the triple zeta valence plus polarization (TZVP) basis set [39] was used for both $\mathrm{Al}$ and $\mathrm{H}$. Harmonic vibrational frequencies were calculated at the same level of theory making use of analytical Hessians to ensure that all the stationary structures have no imaginary frequency, and zeropoint energy (ZPE) corrections were taken into consideration in the evaluation of relative energies. The IR spectra of $\mathrm{Al}_{n} \mathrm{Rh}_{2} \mathrm{H}_{2,4}{ }^{+}(n=10-13)$ complexes were simulated from the harmonic frequencies and intensities.

\section{Results and Discussion}

A mass spectrum in the size range of $\mathrm{Al}_{n} \mathrm{Rh}_{2}{ }^{+}(n=10-13)$, with and without hydrogen, is shown in Fig. 2a. Next to the bare cluster peaks, at an equidistant spacing of two mass units, the hydrogenated complexes can be observed. Although species with one, two and three rhodium dopants were present in the molecular beam, we limit the scope of this paper to the doubly doped ones, and more specifically to the four sizes $n=10,11,12$ and 13. Compared to the singly doped ones, the doubly doped clusters are far more reactive, and compared to the triply doped ones, the double doped clusters are computationally more tractable. The four sizes $\mathrm{Al}_{10-13} \mathrm{Rh}_{2}{ }^{+}$were selected because of their overall greater abundance in the mass spectra, resulting in a high signalto-noise ratio of the infrared multiple photon dissociation spectra. Moreover, these spectra exhibit some remarkable features, as will be discussed in more detail in the analysis of the infrared spectroscopic data.

The fractional distribution of $\mathrm{Al}_{n} \mathrm{Rh}_{2} \mathrm{H}_{2 p}{ }^{+}$complexes $(n=10-13, p=0-4)$, produced at a hydrogen backing pressure of 1900 mbar, can be calculated from the abundances $I$ in the mass spectra as

$\left[\mathrm{Al}_{n} \mathrm{Rh}_{2} \mathrm{H}_{2 p}{ }^{+}\right]_{\text {frac }}=\frac{I\left(\mathrm{Al}_{n} \mathrm{Rh}_{2} \mathrm{H}_{2 p}{ }^{+}\right)}{\sum_{i=0}^{4} I\left(\mathrm{Al}_{n} \mathrm{Rh}_{2} \mathrm{H}_{2 i}{ }^{+}\right)}$.

This quantity is plotted in Fig. $2 \mathrm{~b}$ and provides a qualitative means for assessing cluster reactivity towards hydrogen. For $n=10$, the least reactive species of the series, an approximately equal amount of one and two hydrogen molecules were observed to adsorb onto the cluster. For $n=11,12$, twothirds of the clusters adsorb one $\mathrm{H}_{2}$ and about one-fourth adsorbs a second $\mathrm{H}_{2}$. The last cluster in the series, $\mathrm{Al}_{13} \mathrm{Rh}_{2}{ }^{+}$, predominantly adsorbs a single hydrogen molecule.

Argon is often used as a probe to extract structural information of clusters in the gas phase. Its tendency to bind rather weakly makes that it is often used as a spectator ligand in IRMPD and other action spectroscopic studies [40-42]. Less direct structural evidence can be obtained when only specific constituents or geometrical sites of the cluster are reactive towards argon, e.g. the transition metal (TM) in $\mathrm{TM}$ doped aluminum clusters $(\mathrm{TM}=\mathrm{V}, \mathrm{Cr}, \mathrm{Ti})[43,44]$. To probe the reactivity of the clusters towards argon, the source was cooled down with liquid $\mathrm{N}_{2}$ to $90 \mathrm{~K}$ and a small amount of Ar was mixed into the carrier gas (2\% Ar, 98\% $\mathrm{He})$. The resulting fractional distribution of $\mathrm{Al}_{10-13} \mathrm{Rh}_{2}^{+} \cdot \mathrm{Ar}_{m}$ $(m=0-2)$ complexes is shown in Fig. $2 c$ and, quite serendipitously, manifests a striking similarity between the reactivity of the clusters towards argon and hydrogen: little to no Ar can be attached to $\mathrm{Al}_{10} \mathrm{Rh}_{2}{ }^{+}$, whereas approximately 30-50\% of the $\mathrm{Al}_{11-13} \mathrm{Rh}_{2}{ }^{+}$clusters adsorb a single argon atom. 
Fig. 2 a Mass spectrum of bare (black) and hydrogenated (red) $\mathrm{Al}_{n} \mathrm{Rh}_{m}{ }^{+}$clusters $\left(^{\circ}=\mathrm{Al}_{6-9} \mathrm{Rh}_{3}{ }^{+}, \bullet=\mathrm{Al}_{10-13} \mathrm{Rh}_{2}{ }^{+}\right.$, $\left.*=\mathrm{Al}_{14-17} \mathrm{Rh}^{+}\right)$, b fractional distribution of hydrogenated $\mathrm{Al}_{10-13} \mathrm{Rh}_{2}{ }^{+}$clusters, $\mathbf{c}$ fractional distribution of Ar-tagged $\mathrm{Al}_{10-13} \mathrm{Rh}_{2}{ }^{+}$clusters. The clusters for which IRMPD spectra were measured are designated by red crosses in $\mathbf{a}$

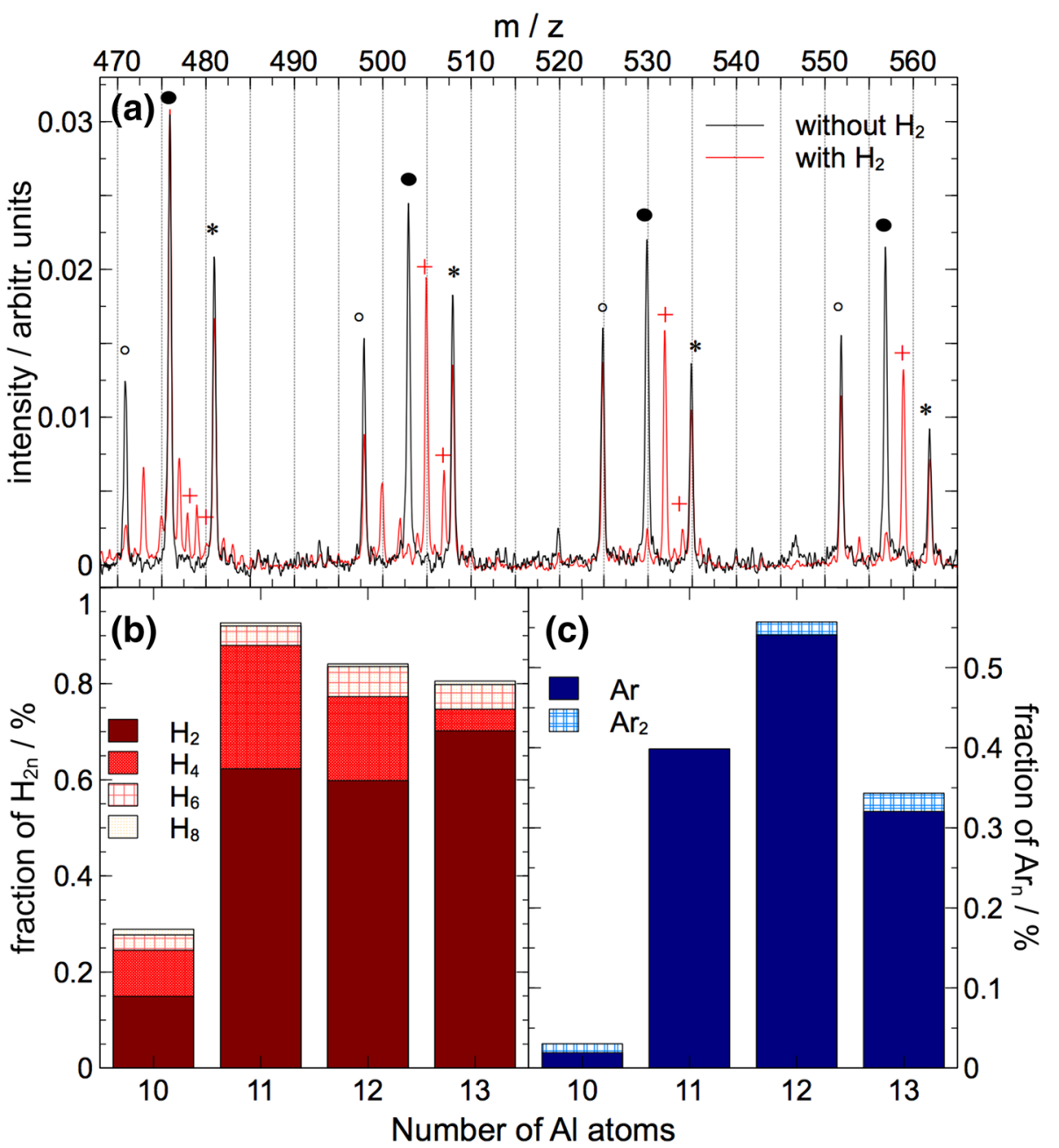

The similarity between the size dependent reactivity towards hydrogen and argon suggests that like for argon, the binding mechanism for hydrogen involves a precursor state, in which the hydrogen is molecularly bound, and which has been observed in the reaction of $\mathrm{TM}_{n}$ clusters with $\mathrm{H}_{2} / \mathrm{D}_{2}$ and $\mathrm{N}_{2}(\mathrm{TM}=\mathrm{Fe}, \mathrm{Co}, \mathrm{Ni}, \mathrm{W})$ [45]. Such a precursor state generally enhances the reactivity compared to the sterically more stringent requirements of direct reactions, essentially giving the hydrogen molecule more time to probe the potential energy landscape for a possible chemisorption/dissociation channel. As almost no argon could be attached to the singly rhodium doped aluminum clusters with the source at $90 \mathrm{~K}$, the existence of a precursor state could explain the increased reactivity of the aluminum clusters with two rhodium dopants. The nature of the cluster-argon interaction will be discussed in the paragraphs containing the computational results.

Previous infrared studies of hydrogenated and deuterated transition metal and transition metal doped aluminum clusters reported absorption bands in the mid-infrared regime, i.e. between 600 and $2600 \mathrm{~cm}^{-1}$ [22, 46, 47]. This broad spectral range can be roughly divided into three regions, each specific to a different type of vibration: the $\mathrm{H}_{2}-$ metal (M) stretch between 600 and $900 \mathrm{~cm}^{-1}$, with the hydrogen molecule molecularly adsorbed; the $\mathrm{H}-\mathrm{M}$ vibrational modes, for which the hydrogen adsorbs dissociatively and can be found in multiple coordination sites (on-top, bridging, threefold coordinated, ...), located between 800 and $2000 \mathrm{~cm}^{-1}$; and the D-D stretching frequency, becoming IR-active only after adsorption, in the higher wavenumber range between 2200 and $2600 \mathrm{~cm}^{-1}$. Noteworthy is the $\mathrm{H}-\mathrm{Al}$ stretching vibrational mode at $1900 \mathrm{~cm}^{-1}$ observed for vanadium doped aluminum clusters [22], evidencing dissociative hydrogenation and spillover from the transition metal dopant to the cluster's aluminum framework. In light of the inertness of pure aluminum (clusters) and the assumed role of the dopant atom as a catalytic center, this is quite remarkable; not only does the relatively strong $\mathrm{H}_{2}$ bond get broken, but the resulting hydrogen atoms move from the dopant to the aluminum.

The IRMPD spectra of $\mathrm{Al}_{n} \mathrm{Rh}_{2} \mathrm{H}_{2 p}{ }^{+}(n=10-13, p=1$, 2 ) are shown in Fig. 3. For $n=10,11$, the most prominent absorption band, for clusters with a single as well as two $\mathrm{H}_{2}$ 

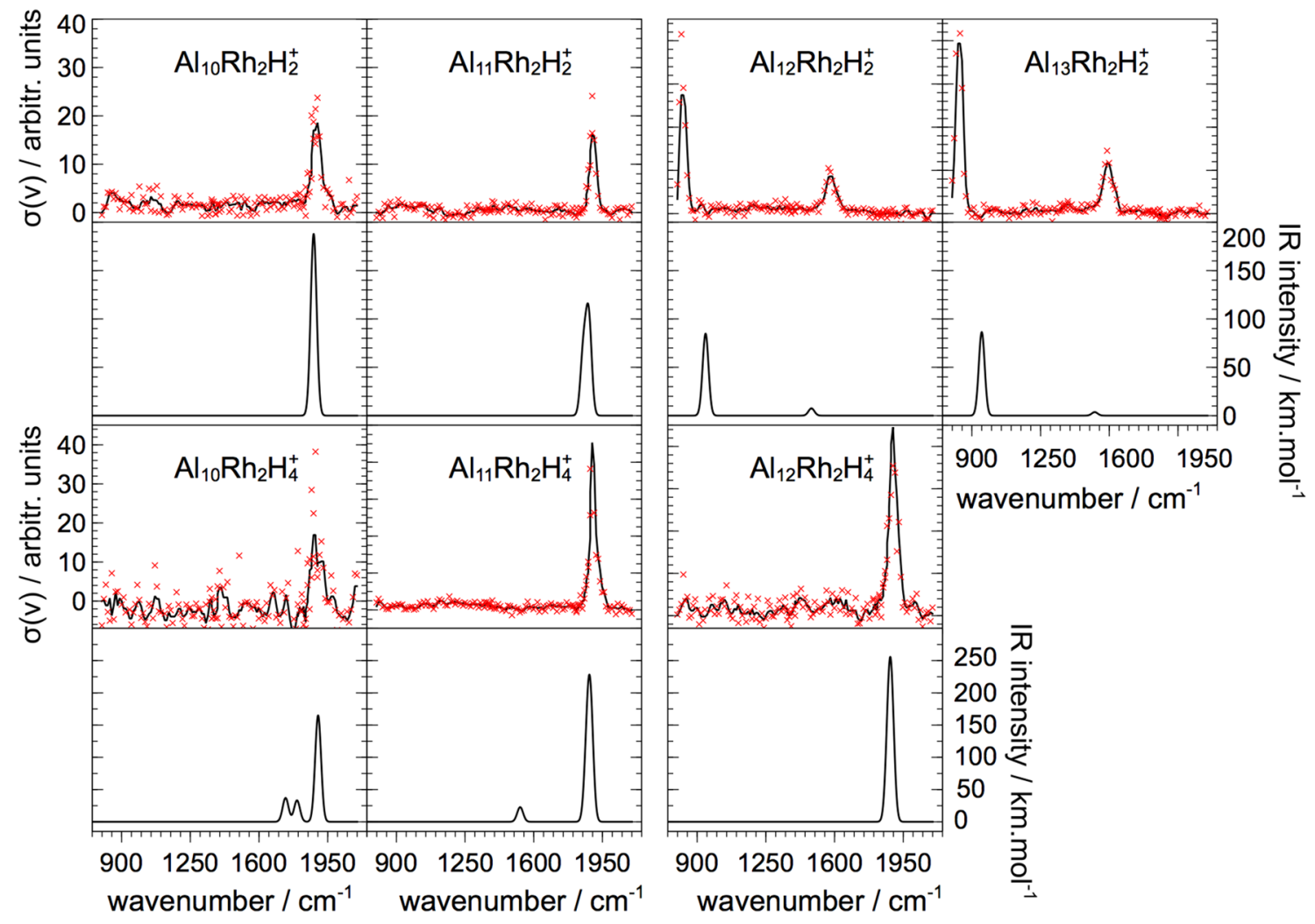

Fig. 3 IRMPD spectra (top panels) of $\mathrm{Al}_{n} \mathrm{Rh}_{2} \mathrm{H}_{2 p}{ }^{+}$complexes $(n=10-13, p=1,2)$ compared to calculated spectra (bottom panels) of the most stable complexes. The crosses are the original data points, while the full lines correspond to three-point running averages

molecules adsorbed, is located at approximately $1900 \mathrm{~cm}^{-1}$ and suggests dissociative adsorption of the hydrogen, bound in an on-top aluminum coordination site. For $n=12$, 13, however, two absorption bands can be distinguished if one hydrogen molecule is adsorbed onto the cluster: one between 800 and $850 \mathrm{~cm}^{-1}$, and another between 1550 and $1600 \mathrm{~cm}^{-1}$. For $\mathrm{Al}_{12} \mathrm{Rh}_{2} \mathrm{H}_{2}{ }^{+}$, both bands disappear upon the addition of a second hydrogen, giving way to the sole feature around $1900 \mathrm{~cm}^{-1}$ as seen for $\mathrm{Al}_{10,11} \mathrm{Rh}_{2} \mathrm{H}_{2,4}{ }^{+}$.

To assign these absorption bands to different hydrogen binding geometries, the infrared spectra of several isomers for each cluster size were calculated and compared with the experimental spectra. Figure 4 shows the tentative ground state structures of bare and hydrogenated $\mathrm{Al}_{10-13} \mathrm{Rh}_{2}{ }^{+}$. The calculated spectra of these lowest energy isomers are plotted (without scaling factor) beneath the experimental spectra in Fig. 3. Structures and infrared spectra for other isomers can be found in the supporting information (SI). All calculated low energy structures are, unless mentioned explicitly, found to have the lowest possible spin configuration (i.e. either singlet or doublet). It is important to note that for the hydrogenated species, the lowest energy structure might not be present in the molecular beam due to activation barriers in the potential energy surface of the reaction from the bare to hydrogenated cluster. Moreover, because there are only minor differences between the calculated infrared spectra of the isomers and the spectrum of the tentative ground state structure, it is impossible to identify the structure present in the experiment unambiguously. What can be learned in this comparison, however, is the general nature of the hydrogen binding to the cluster (molecular/dissociative, spillover from/poisoning of the dopant). For $n=11,12$, inspection of the calculated normal modes shows that the resonances at $1900 \mathrm{~cm}^{-1}$ correspond indeed to the $\mathrm{Al}-\mathrm{H}$ stretching frequency of a hydrogen atom bound to a single $\mathrm{Al}$ atom (on-top), i.e. at least one of the hydrogen molecules dissociates upon adsorption and the hydrogen atoms spill over to the aluminum. Although all calculated isomers within $0.5 \mathrm{eV}$ of the ground state structure have both molecules adsorbed dissociatively, it could be that these structures are kinetically unreachable and therefore one cannot dismiss the possibility that the second hydrogen is bound molecularly, with IR resonances outside the range of the measurement. The small features around $1750 \mathrm{~cm}^{-1}$ in the theoretical infrared spectrum of $\mathrm{Al}_{10} \mathrm{Rh}_{2} \mathrm{H}_{4}{ }^{+}$correspond to a hydrogen atom bound to a rhodium atom, but are not clearly visible in the experimental spectrum. 
Fig. 4 Putative ground state structures of bare (top row) and hydrogenated (middle and bottom row) $\mathrm{Al}_{10-13} \mathrm{Rh}_{2}{ }^{+}$ (pearl blue $=$ aluminum, dark green $=$ rhodium, white $=$ hydrogen). For $n=12,13$, the lowest energy isomers contain molecularly adsorbed $\mathrm{H}_{2}$. For all other clusters, the hydrogen molecules dissociate upon adsorption
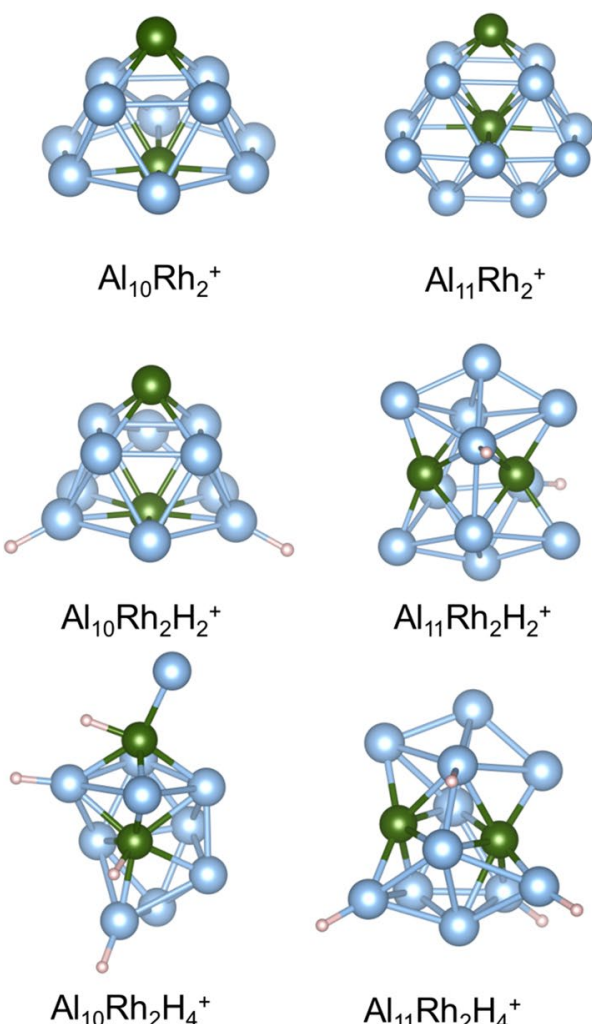

$\mathrm{Al}_{11} \mathrm{Rh}_{2} \mathrm{H}_{2}{ }^{+}$

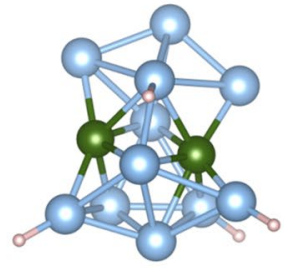

$\mathrm{Al}_{11} \mathrm{Rh}_{2} \mathrm{H}_{4}^{+}$

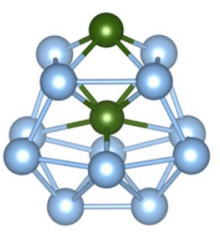

$\mathrm{Al}_{12} \mathrm{Rh}_{2}{ }^{+}$

8

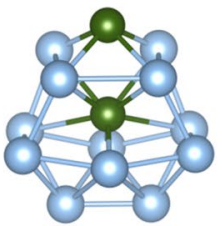

$\mathrm{Al}_{12} \mathrm{Rh}_{2} \mathrm{H}_{2}{ }^{+}$

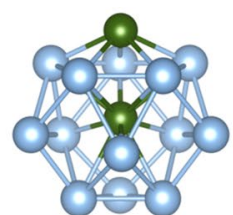

$\mathrm{Al}_{13} \mathrm{Rh}_{2}{ }^{+}$

$0=0$

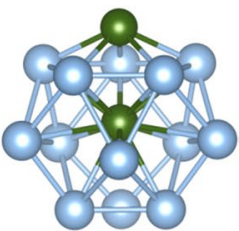

$\mathrm{Al}_{13} \mathrm{Rh}_{2} \mathrm{H}_{2}{ }^{+}$
For $n=12,13$, the calculations reveal that the absorption bands around $800 \mathrm{~cm}^{-1}$ and $1600 \mathrm{~cm}^{-1}$ are due to the stretching and bending mode of the molecularly bound hydrogen respectively. The band at $1900 \mathrm{~cm}^{-1}$ in the calculated IRMPD spectra of $\mathrm{Al}_{12} \mathrm{Rh}_{2} \mathrm{H}_{4}{ }^{+}$corresponds again to on-top $\mathrm{H}-\mathrm{Al}$ stretches. The same caveat as for $n=10,11$ applies to $\mathrm{Al}_{12} \mathrm{Rh}_{2} \mathrm{H}_{4}{ }^{+}$: although both the absence of other bands in the experimental spectrum and the calculated IRMPD spectra of the low energy isomers (SI) strongly suggest that the second $\mathrm{H}_{2}$ molecule adsorbs dissociatively as well, the possibility that the second hydrogen is bound molecularly and has resonances outside the range of the measurement remains.

Table 1 contains the calculated binding energies for argon $\left(B E(\mathrm{Ar})=E\left(\mathrm{Al}_{10-13} \mathrm{Rh}_{2}{ }^{+} \cdot \mathrm{Ar}\right)-E\left(\mathrm{Al}_{10-13} \mathrm{Rh}_{2}{ }^{+}\right)-\right.$ $E(\mathrm{Ar}))$, hydrogen in molecular form $\left(B E\left(\mathrm{H}_{2}\right)=E\left(\mathrm{Al}_{10-13}\right.\right.$ $\left.\left.\mathrm{Rh}_{2}{ }^{+} \cdot \mathrm{H}_{2}\right)-E\left(\mathrm{Al}_{10-13} \mathrm{Rh}_{2}{ }^{+}\right)-E\left(\mathrm{H}_{2}\right)\right)$, hydrogen in atomic form $\left(B E(2 \mathrm{H})=E\left(\mathrm{Al}_{10-13} \mathrm{Rh}_{2} \mathrm{H}_{2}^{+}\right)-E\left(\mathrm{Al}_{10-13} \mathrm{Rh}_{2}{ }^{+}\right)-E\left(\mathrm{H}_{2}\right)\right)$, and for a second, dissociatively bound $\mathrm{H}_{2}$ molecule $(B E(4 \mathrm{H}-2 \mathrm{H})$ $\left.=E\left(\mathrm{Al}_{10-13} \mathrm{Rh}_{2} \mathrm{H}_{4}{ }^{+}\right)-E\left(\mathrm{Al}_{10-13} \mathrm{Rh}_{2} \mathrm{H}_{2}^{+}\right)-E\left(\mathrm{H}_{2}\right)\right) . B E\left(\mathrm{H}_{2}\right)$ is obtained by scanning the surface of the bare cluster for the most stable $\mathrm{H}_{2}$ binding position, in each case being ontop of the outermost rhodium atom. The values in brackets are the binding energies obtained at the PBE-GD3BJ level to account for dispersions forces. Although dispersion increases the BEs by $0.04-0.10 \mathrm{eV}$, its inclusion neither alters the size dependence nor the preference for molecular or atomic adsorption of hydrogen. In the last three columns, the $\mathrm{H}-\mathrm{H}$ bond distance $d(\mathrm{H}-\mathrm{H})$ for the molecularly bound hydrogen, the natural population analysis (NPA) negative charge $q(\mathrm{Rh})$ on the top rhodium atom, and the vertical and adiabatic ionization $E_{\mathrm{VIE} / \mathrm{AIE}}$ are tabulated. These values with inclusion of dispersion barely changed and are therefore omitted. The size-dependence of the calculated $\mathrm{Ar}$ and $\mathrm{H}_{2}$ binding energies are clearly correlated with the observed abundances in Fig. 1b, c. For the Ar adsorption, the binding energy also correlates with $q(\mathrm{Rh})$ and together with the large distance between the top rhodium and the argon ( $>4 \AA$ for all sizes) suggests that the bond is electrostatic in nature.
Table 1 Calculated Ar, $\mathrm{H}_{2}, 2 \mathrm{H}$ and $4 \mathrm{H}-2 \mathrm{H}$ binding energies of $\mathrm{Al}_{10-13} \mathrm{Rh}_{2}{ }^{+}, d\left(\mathrm{H}_{2}\right)$, the charge $q(\mathrm{Rh})$ on the top rhodium atom, and $E_{\mathrm{VIE} / \mathrm{AIE}}$ at the PBE and PBE-GJ3BJ (in brackets) level

\begin{tabular}{llllllll}
\hline$n$ & $B E(\mathrm{Ar})(\mathrm{eV})$ & $B E\left(\mathrm{H}_{2}\right)(\mathrm{eV})$ & $B E(2 \mathrm{H})(\mathrm{eV})$ & $B E(4 \mathrm{H}-2 \mathrm{H})(\mathrm{eV})$ & $d(\mathrm{H}-\mathrm{H})(\mathrm{A})$ & $q(\mathrm{Rh})(e)$ & $\mathrm{E}_{\mathrm{VIE} / \mathrm{AIE}}(\mathrm{eV})$ \\
\hline 10 & $0.07(0.12)$ & $0.20(0.24)$ & $0.72(0.79)$ & $0.88(0.98)$ & 0.78 & 0.91 & $9.56 / 9.48$ \\
11 & $0.16(0.22)$ & $0.67(0.73)$ & $0.79(0.87)$ & $0.71(0.82)$ & 0.85 & 1.07 & $9.77 / 9.63$ \\
12 & $0.18(0.25)$ & $0.70(0.77)$ & $0.62(0.74)$ & $0.48(0.56)$ & 0.85 & 1.31 & $9.60 / 9.50$ \\
13 & $0.17(0.24)$ & $0.70(0.77)$ & $0.39(0.46)$ & & 0.86 & 1.04 & $9.44 / 9.35$ \\
\hline
\end{tabular}


For $n=11,12$ and 13, the $\mathrm{H}-\mathrm{H}$ distance in the molecularly bound state is significantly elongated compared to the free $\mathrm{H}_{2}$ molecule $\left(d_{\text {exp }}(\mathrm{H}-\mathrm{H})=0.74 \AA\right.$ [48], $\left.d_{\text {calc }}(\mathrm{H}-\mathrm{H})=0.76 \AA\right)$, which, together with the relatively high $B E\left(\mathrm{H}_{2}\right)$, is suggestive of the formation of a Kubas complex [26].

The binding energies for molecular versus dissociative adsorption, $B E\left(\mathrm{H}_{2}\right)$ and $B E(2 \mathrm{H})$, demonstrate that for $\mathrm{Al}_{12,13} \mathrm{Rh}_{2}{ }^{+}$, as opposed to $\mathrm{Al}_{10,11} \mathrm{Rh}_{2}{ }^{+}$, the molecularly bound state is lower in energy than that of the lowest energy structure in which the hydrogen binds dissociatively. This points in the direction of a thermodynamically and not kinetically controlled reaction, in stark contrast with the observed reactivity pattern of vanadium doped aluminum clusters [22]. In ref. [22], lower abundancies in the mass spectrum were explained instead by activation barriers in the hydrogenation pathway, which were attributed to unfavorable orbital overlap between the cluster and $\mathrm{H}_{2}$ orbitals. The left side of Fig. 5 shows the highest occupied molecular orbitals (HOMO) of the $\mathrm{Al}_{10-13} \mathrm{Rh}_{2}{ }^{+}$clusters. The HOMOs of the clusters after the initial step in the hydrogenation pathway, with the hydrogen molecule bound molecularly, are shown in the right side of Fig. 5.

Focusing on the structural motif which initiates the reaction with the hydrogen molecule, i.e. the top pyramidal structure with one of the rhodium atoms at the apex, one can see that the HOMOs for $n=11,12$ and 13 are very similar to each other; they are formed by hybridization of the $d_{x z} / d_{y z}$ atomic orbitals of the top rhodium atom with the delocalized orbitals deriving from the aluminum $s$ - and $p$-electrons, and have a significant amplitude in the direction perpendicular to the basal plane of the pyramidal motif. The spin-up and spin-down HOMOs for $n=10$, on the other hand, do not have obvious $d_{x z} / d_{y z}$ character. Instead, they derive from the $d_{x y} / d_{x^{2}-y^{2}}$ orbitals of the top rhodium atom, which have less amplitude in the direction of the $z$-axis. As can be seen on the right side of Fig. 5, for $n=11,12,13$, the HOMOs of the bare clusters give rise to the formation of a Kubascomplex [26], in which charge is transferred from the cluster to the $\sigma^{*}$ anti-bonding orbital of the hydrogen molecule. For $n=10$, on the contrary, inspection of the orbitals of the hydrogen-cluster complex bore no evidence of the formation of a Kubas complex.

If charge transfer plays a dominant role in the activation one may expect correlations between reaction behavior and the ionization energetics of the cluster. In fact, for the molecular chemisorption of hydrogen on small, anionic titanium clusters that has been experimentally investigated by Burkart et al. [49] it is argued that molecular adsorption is favored over dissociative adsorption because the electron detachment energy is large for small clusters and therefore the charge transfer to hydrogen small. Similarly, the molecular adsorption of hydrogen on $\mathrm{Ni}^{+}$, studied computationally by Niu et al. [25], was attributed to the cationic nature of the nickel ion, which impedes charge transfer to the hydrogen
Fig. 5 Highest occupied molecular orbitals (HOMOs) of the $\mathrm{Al}_{10-13} \mathrm{Rh}_{2}{ }^{+}$clusters without (left) and with (right) hydrogen adsorbed molecularly. For $n=10,12$, both spin up and spin down HOMOs are shown. The isosurfaces correspond to an electronic charge density of $0.02 \mathrm{e} / \AA^{3}$
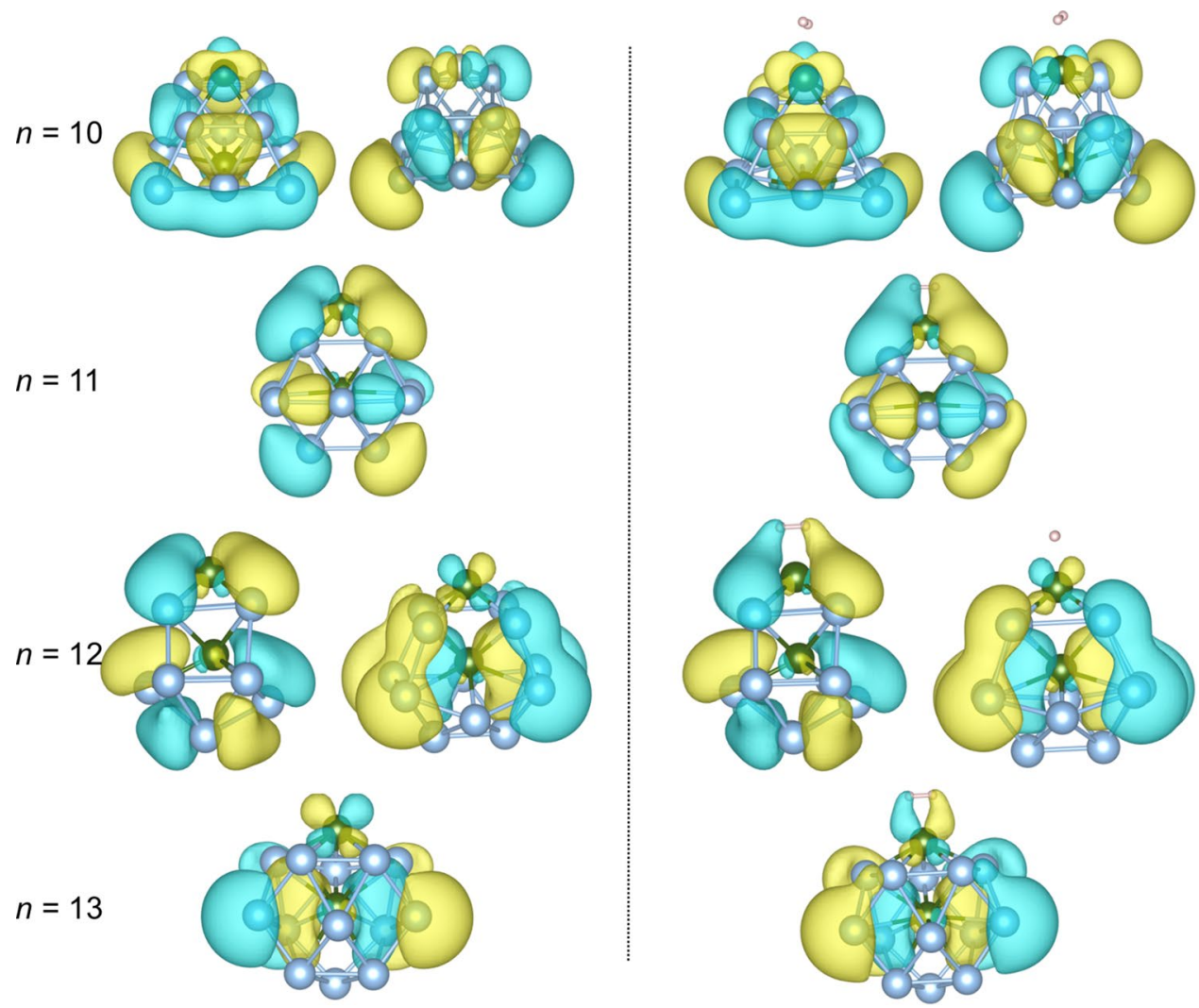
molecule. As can be seen from the $E_{\mathrm{VIE} / \mathrm{AIE}}$ calculations in Table 1, however, there is no simple correlation between the calculated ionization energies and the molecular hydrogen binding energies, nor an anti-correlation with the dissociative binding energies. A different, possible explanation as to why the difference in binding geometry of the first hydrogen molecule between $\mathrm{Al}_{11} \mathrm{Rh}_{2}{ }^{+}$and $\mathrm{Al}_{12,13} \mathrm{Rh}_{2}{ }^{+}$can be found in the delocalized part of the HOMOs. For $n=11$, the HOMO close to the hydrogen is delocalized and hence there is a stronger electron-electron repulsion with the electrons in the $\sigma$ bonding orbital of $\mathrm{H}_{2}$, which destabilizes the Kubas complex. For $n=12$, only the spin-up orbital is delocalized in the neighborhood of the hydrogen binding site and so the electrostatic repulsion is more moderate. For $n=13$, the binding occurs mainly through the localized rhodium $d_{x z}$ -orbital part of the HOMO, whereas the delocalized part of the HOMO is more isolated from the $\sigma$ bonding orbital of the hydrogen molecule. Table 1 shows that it is indeed energetically more favorable (by $0.12 \mathrm{eV}$ ) to bind the hydrogen molecule dissociatively for $n=11$. For $\mathrm{Al}_{12} \mathrm{Rh}_{2}{ }^{+}$, however, both modes are almost iso-energetic, with the molecular adsorption mode slightly lower in energy. The low energy isomers in the supporting information (Fig. S2/S4) suggest a competitive nature of the adsorption mode for $n=12$ as well, alternating between dissociative and molecular adsorption. For $n=13, \mathrm{H}_{2}$ binds molecularly for all low energy isomers, and the calculated energy difference between the two modes is substantially larger $(0.31 \mathrm{eV})$.

\section{Conclusion}

In summary, the interaction of hydrogen with $\mathrm{Al}_{n} \mathrm{Rh}_{2}{ }^{+}$ $(n=10-13)$ clusters was studied experimentally by mass spectrometry and infrared multiple photon dissociation spectroscopy. The similarity between the abundance pattern of hydrogenated and argon-tagged clusters suggests the existence of a precursor state which could account for the increased reactivity of $\mathrm{Al}_{11-13} \mathrm{Rh}_{2}{ }^{+}$as compared to $\mathrm{Al}_{10} \mathrm{Rh}_{2}{ }^{+}$. Comparison of the infrared absorption bands with density functional theory calculations shows that for $n=10$ and 11 , the first $\mathrm{H}_{2}$ binds dissociatively, whereas for $n=12,13$, it binds molecularly. Upon adsorption of a second hydrogen molecule, the experimental spectra only show vibrational modes corresponding to hydrogen bound dissociatively mainly in on-top aluminum positions. This observation strongly suggests hydrogen spillover from the rhodium dopant to the aluminum atoms, which is in agreement with the theoretical predictions. Calculations of the hydrogen binding energy suggest that the molecular adsorption for $n=12$ and 13 is not kinetically but thermodynamically controlled, i.e. due to a higher binding energy of the molecularly adsorbed hydrogen-cluster complex. Inspection of the highest occupied molecular orbitals shows that the hydrogen molecule initially forms a strongly-bound Kubas complex with the $\mathrm{Al}_{11-13} \mathrm{Rh}_{2}{ }^{+}$clusters, and not with $\mathrm{Al}_{10} \mathrm{Rh}_{2}{ }^{+}$. Although few-atom clusters in the gas phase differ significantly from the nanomaterials studied for applications in hydrogen storage, the size-dependency of the adsorption mode of hydrogen onto doubly rhodium doped aluminum clusters and the observed spillover from the dopants to the aluminum atoms could be of aid in the design of novel hydrogen storage materials.

Acknowledgements This work is supported by the KU Leuven Research Council (GOA/14/007). J.V. would like to thank the FWOResearch Foundation Flanders for a PhD fellowship. P.F. acknowledges CONICyT for a Becas Chile scholarship. A.F. thanks the Deutsche Forschungsgemeinschaft for a Heisenberg grant (FI 893/5).

\section{References}

1. Dawson VP, Bowles MD (2004) Taming Liquid Hydrogen: The Centaur Upper Stage Rocket, 1958-2002. Diane Publishing Co

2. Durbin DJ, Malardier-Jugroot C (2013) Review of hydrogen storage techniques for on board vehicle applications. Int $\mathbf{J}$ Hydrogen Energy 38:14595-14617. https://doi.org/10.1016/j. ijhydene.2013.07.058

3. Orimo S-I, Nakamori Y, Eliseo JR et al (2007) Complex hydrides for hydrogen storage. Chem Rev 107:4111-4132. https://doi. org/10.1021/cr0501846

4. Ley MB, Jepsen LH, Lee Y et al (2014) Complex hydrides for hydrogen storage-new perspectives. Mater Today 17:122-128. https://doi.org/10.1016/j.mattod.2014.02.013

5. Chaudhuri S, Graetz J, Ignatov A et al (2006) Understanding the role of $\mathrm{Ti}$ in reversible hydrogen storage as sodium alanate: a combined experimental and density functional theoretical approach. J Am Chem Soc 128:11404-11415. https://doi.org/10.1021/ ja060437s

6. Baldé CP, Hereijgers BPC, Bitter JH, de Jong KP (2008) Sodium alanate nanoparticles - linking size to hydrogen storage properties. J Am Chem Soc 130:6761-6765. https://doi.org/10.1021/ ja710667v

7. Zaera F (2013) Nanostructured materials for applications in heterogeneous catalysis. Chem Soc Rev 42:2746-2762. https://doi. org/10.1039/C2CS35261C

8. Jena P (2011) Materials for hydrogen storage: past, present, and future. J Phys Chem Lett 2:206-211. https://doi.org/10.1021/ jz1015372

9. Tyo EC, Vajda S (2015) Catalysis by clusters with precise numbers of atoms. Nat Nanotechnol 10:577-588. https://doi.org/10.1038/ nnano.2015.140

10. Janssens E, Le HT, Lievens P (2015) Adsorption of propene on neutral gold clusters in the gas phase. Chem-A Eur J 21:1525615262. https://doi.org/10.1002/chem.201500523

11. Lang SM, Bernhardt TM (2012) Gas phase metal cluster model systems for heterogeneous catalysis. Phys Chem Chem Phys 14:9255-9269. https://doi.org/10.1039/c2cp40660h

12. Schwarz H (2015) Doping effects in cluster-mediated bond activation. Angew Chem Int Ed 54:10090-10100. https://doi. org/10.1002/anie.201500649

13. Sanchez A, Abbet S, Heiz U, et al (1999) When Gold Is Not Noble: Nanoscale Gold Catalysts. J Phys Chem A 103:9573-9578. https://doi.org/10.1021/jp9935992 
14. Ferrari P, Molina LM, Kaydashev VE et al (2016) Controlling the adsorption of carbon monoxide on platinum clusters by dopantinduced electronic structure modification. Angew Chem Int Ed 128:11225-11229. https://doi.org/10.1002/anie.201604269

15. Kiohara VO, Carvalho EFV, Paschoal CWA et al (2013) DFT and CCSD (T) electronic properties and structures of aluminum clusters: $\mathrm{Al}_{n x}(n=1-9, x=0, \pm 1)$. Chem Phys Lett 568-569:42-48. https://doi.org/10.1016/j.cplett.2013.03.005

16. Upton TH, Cox DM, Kaldor A (1987) Activation and chemisorption of hydrogen on aluminum clusters. In: Jena P, Rao BK, Khanna SN (eds) Physics and chemistry of small clusters. Springer, New York, pp 755-768. https://doi. org/10.1007/978-1-4757-0357-3_100

17. Pino I, Kroes GJ, Van Hemert MC (2010) Hydrogen dissociation on small aluminum clusters. J Chem Phys 133:184304. https://doi. org/10.1063/1.3502493

18. Zhang F, Wang Y, Chou MY (2012) Hydrogen interaction with the Al surface promoted by subsurface alloying with transition metals. J Phys Chem C 116:18663-18668. https://doi.org/10.1021/ jp306377j

19. Chopra IS, Chaudhuri S, Veyan JF, Chabal YJ (2011) Turning aluminium into a noble-metal-like catalyst for low-temperature activation of molecular hydrogen. Nat Mater 10:986-986. https:// doi.org/10.1038/nmat3174

20. Menezes WJC, Knickelbein MB (1991) Bimetallic clusters of cobalt and aluminum: ionization potentials versus reactivity, and the importance of geometric structure. Chem Phys Lett 183:357362. https://doi.org/10.1016/0009-2614(91)90393-N

21. Nonose S, Sone Y, Onodera K et al (1989) Reactivity study of alloy clusters made of aluminum and some transition metals with hydrogen. Chem Phys Lett 164:427-432. https://doi. org/10.1016/0009-2614(89)85232-7

22. Vanbuel J, Fernandez EM, Ferrari P et al (2017) Hydrogen chemisorption on singly vanadium-doped aluminum clusters. Chem-A Eur J 23:1-7. https://doi.org/10.1002/chem.201704361

23. Charkin OP, Mikhailin AA, Klimenko NM (2013) Theoretical modeling of elementary reactions of dissociative addition of an $\mathrm{H}_{2}$ molecule to aluminum clusters $\mathrm{MAl}_{12}$ doped with early $3 \mathrm{~d}$ and $4 \mathrm{~d}$ transition metal atoms. Russ J Inorg Chem 58:1479-1488. https:// doi.org/10.1134/S0036023613120073

24. Pramann A, Nakajima A, Kaya K (2001) Photoelectron spectroscopy of bimetallic aluminum cobalt cluster anions: comparison of electronic structure and hydrogen chemisorption rates. J Chem Phys 115:5404-5410. https://doi.org/10.1063/1.1394944

25. Niu J, Rao BK, Jena P (1992) Binding of hydrogen molecules by a transition-metal ion. Phys Rev B 68:2277-2281. https://doi. org/10.1103/PhysRevLett.68.2277

26. Kubas GJ (2007) Fundamentals of H2 binding and reactivity on transition metals underlying hydrogenase function and $\mathrm{H} 2$ production and storage. Chem Rev 107:4152-4205. https://doi. org/10.1021/cr050197j

27. Truong NX, Haertelt M, Jaeger BKAA. et al (2016) Characterization of neutral boron-silicon clusters using infrared spectroscopy: the case of $\mathrm{Si}_{6} \mathrm{~B}$. Int J Mass Spectrom 395:1-6. https://doi. org/10.1016/j.ijms.2015.11.006

28. Schöllkopf W, Gewinner S, Erlebach W et al (2014) The new IR FEL facility at the Fritz-Haber-Institut in Berlin. In: Proceedings of FEL 2014, Basel, WEB04, pp 629-634

29. Schöllkopf W, Gewinner S, Junkes H et al (2015) The new IR and THz FEL facility at the Fritz Haber Institute in Berlin. Proc SPIE 9512:95121L. https://doi.org/10.1117/12.2182284

30. Nesbitt DJ, Field RW (1996) Vibrational energy flow in highly excited molecules: role of intramolecular vibrational redistribution. J Phys Chem 100:12735-12756. https://doi.org/10.1021/ jp960698w
31. Herzberg G (1969) Dissociation energy and ionization potential of molecular hydrogen. Phys Rev Lett 23:1081-1083. https://doi. org/10.1103/PhysRevLett.23.1081

32. Kawamura H, Kumar V, Sun Q, Kawazoe Y (2001) Magic behavior and bonding nature in hydrogenated aluminum clusters. Mater Chem Phys 115:612-617. https://doi.org/10.1016/j. matchemphys.2009.01.025

33. Wang H, Wang Y, Lv J et al (2016) CALYPSO structure prediction method and its wide application. Comput Mater Sci 112:406415. https://doi.org/10.1016/j.commatsci.2015.09.037

34. Perdew JP, Burke K, Ernzerhof M (1996) Generalized gradient approximation made simple. Phys Rev Lett 77:3865-3868. https:// doi.org/10.1103/PhysRevLett.77.3865

35. Petrie S, Stranger R (2004) DFT and metal-metal bonding: a dysfunctional treatment for multiply charged complexes? Inorg Chem 43:2597-2610. https://doi.org/10.1021/ic034525e

36. Liu Y, Zhang J, Li J et al (2016) Hydrogen, oxygen and nitrogen adsorption on $\mathrm{Rh}_{n-1} \mathrm{X}(n=2-5, \mathrm{X}=3 \mathrm{~d}, 4 \mathrm{~d}$ atoms) clusters: a DFT study. Comput Theor Chem 1085:56-65. https://doi.org/10.1016/j. comptc.2016.04.008

37. Grimme S, Ehrlich S, Goerigk L (2011) Effect of the damping function in dispersion corrected density functional theory. J Comput Chem 32:1456-1465. https://doi.org/10.1002/jcc

38. Igel-Mann G, Stoll H, Preuss H (1988) Pseudopotentials for main group elements (IIIa through VIIa). Mol Phys 65:1321-1328. https://doi.org/10.1080/00268978800101811

39. Schäfer A, Huber C, Ahlrichs R et al (1994) Fully optimized contracted Gaussian basis sets of triple zeta valence quality for atoms $\mathrm{Li}$ to $\mathrm{Kr}$ fully optimized contracted Gaussian basis sets of triple zeta valence quality for atoms Li to Kr. J Chem Phys 100:5829/7. https://doi.org/10.1063/1.467146

40. Shayeghi A, Johnston RL, Rayner DM et al (2015) The nature of bonding between argon and mixed gold-silver trimers. Angew Chem 54:10675-10680. https://doi.org/10.1002/anie.201503845

41. Gehrke R, Gruene P, Fielicke A et al (2013) Nature of Ar bonding to small $\mathrm{Co}_{\mathrm{n}}{ }^{+}$clusters and its effect on the structure determination by far-infrared absorption spectroscopy. J Chem Phys 130:34306. https://doi.org/10.1063/1.3058637

42. Janssens E, Gruene P, Meijer G et al (2007) Argon physisorption as structural probe for endohedrally doped silicon clusters. Phys Rev Lett 99:1-4. https://doi.org/10.1103/PhysRevLett.99.063401

43. Lang SM, Claes P, Neukermans S, Janssens E (2011) Cage structure formation of singly doped aluminum cluster cations $\mathrm{Al}_{n} \mathrm{TM}^{+}$ ( $\mathrm{TM}=\mathrm{Ti}, \mathrm{V}, \mathrm{Cr}$ ). J Am Soc Mass Spectrom 22:1508-1514. https:// doi.org/10.1007/s 13361-011-0181-1

44. Fernández EM, Vega A, Balbás LC (2013) Theoretical study of $\mathrm{Al}_{n} \mathrm{~V}^{+}$clusters and their interaction with Ar. J Chem Phys 139:214305. https://doi.org/10.1063/1.4834595

45. Knickelbein MB (1999) Reactions of transition metal clusters with small molecules. Annu Rev Phys Chem 50:79-115. https://doi. org/10.1146/annurev.physchem.50.1.79

46. Swart I, Gruene P, Fielicke A et al (2008) Molecular adsorption of $\mathrm{H}_{2}$ on small cationic nickel clusters. Phys Chem Chem Phys 10:5743-5745. https://doi.org/10.1039/b807313a

47. Swart I, de Groot FMF, Weckhuysen BM et al (2008) $\mathrm{H}_{2}$ adsorption on $3 \mathrm{~d}$ transition metal clusters: a combined infrared spectroscopy and density functional study. J Phys Chem A 112:11391149. https://doi.org/10.1021/jp076702t

48. Huber K-P (2013) Molecular spectra and molecular structure: IV. Constants of diatomic molecules. Springer, New York. https://doi. org/10.1007/978-1-4757-0961-2

49. Burkart S, Blessing N, Gantefor G (1999) Indication of a sizedependent transition from molecular to dissociative chemisorption on clusters. Phys Rev B 60:15639-15642. https://doi.org/10.1103/ PhysRevB.60.15639 